\title{
SUPERCRITICAL ELLIPTIC EQUATIONS
}

\author{
MELANIE RUPFLIN AND MICHAEL STRUWE
}

\begin{abstract}
For an elliptic model equation with supercritical power non-linearity we give a complete description of radial solutions and discuss self-similar blowup solutions.
\end{abstract}

\section{INTRODUCTION}

Not much is known about non-trivial solutions $u$ to the equation

$$
-\Delta u=|u|^{p-2} u \text { in } \Omega
$$

on a smoothly bounded domain $\Omega \subset \mathbb{R}^{n}$ when $n \geq 3$ and when the exponent $p$ in the nonlinear term is in the "super-critical" range $p>2^{*}=\frac{2 n}{n-2}$.

Here, in a first attempt to understand this equation more thoroughly, we focus on solutions of (1.1) on $\mathbb{R}^{n} \backslash\{0\}$ which either are radially symmetric or self-similar in the sense that $u=u_{R}$ for all $R>0$, where

$$
u_{R}(x)=R^{a} u(R x), R>0,
$$

with $a:=\frac{2}{p-2}$. Note that this scaling of a solution $u$ of equation (1.1) on $\mathbb{R}^{n} \backslash\{0\}$ again produces a solution $u_{R}$ to (1.1), and $u=u_{R}$ for all $R>0$ if and only if

$$
u(x)=|x|^{-a} v\left(\frac{x}{|x|}\right), x \neq 0,
$$

where $v$ solves the equation

$$
-\Delta_{S^{n-1}} v+b \cdot v=|v|^{p-2} v \text { on } S^{n-1}
$$

with $b:=a(n-2-a)$. Such solutions also might arise from blow-up of singular solutions of (1.1) on a domain $\Omega \subset \mathbb{R}^{n}$ which are stationary in the sense of Pacard [6].

Conversely, if $p>2^{*}$ any solution $v \neq 0$ to (1.4) of class $H^{1} \cap L^{p}$ via (1.3) induces a solution $u \in H_{l o c}^{1} \cap L_{l o c}^{p}\left(\mathbb{R}^{n}\right)$ of (1.1) blowing up at the origin. Equation (1.4) is similar to equation (1.1), but - unless $p=2^{+}:=\frac{2(n-1)}{n-3}$, the critical Sobolev exponent in $n-1$ dimensions - no longer has any of the invariance properties of the latter. When $p \leq 2^{+}$any solution $v$ to (1.4) of class $H^{1}$ is smooth, and solutions $v>0$ have been classified completely. In contrast, there is a vast abundance of sign-changing self-similar solutions; see Section 3 . When $p>2^{+}$we expect equation (1.4) to admit also singular solutions $0<v \in H^{1} \cap L^{p}$, inducing solutions $u \in H^{1}$ of (1.1) blowing up on a higher-dimensional singular set. In a sequel [8] to this paper we plan to study this latter case more closely.

Date: August 30, 2012. 
Our results thus are most conclusive for solutions $u>0$ and may be summarized, as follows. On the one hand, in Theorem 2.1 we characterize the self-similar solution

$$
u^{*}(r)=b^{\frac{1}{p-2}} r^{-a} \in H_{l o c}^{1} \cap L_{l o c}^{p}\left(\mathbb{R}^{n}\right)
$$

as the strong limit $u^{*}=\lim _{R \rightarrow \infty} u_{R}$ of rescalings of any smooth, radial entire solution $u$ of $(1.1)$ with $u(0)>0$. On the other hand, as a consequence of a result of Gidas and Spruck [4], for mildly super-critical exponents $2^{*}<p<2^{+}$the solution $u^{*}$ also is the unique positive, self-similar solution of (1.1) of class $H_{l o c}^{1} \cap L_{l o c}^{p}$ on $\mathbb{R}^{n}$.

Moreover, for arbitrary $p>2^{*}$ the function $u^{*}$ is the only radially symmetric solution of (1.1) with a mild singularity at $r=0$; indeed, by Theorems 2.1 and 2.3 any radial solution $u \neq \pm u^{*}$ which is singular at $r=0$ changes sign infinitely often and blows up at the origin with a predetermined rate which, in particular, implies that $u \notin H_{l o c}^{1}$.

\section{RADiAL SOLUTIONS}

For any dimension $n \geq 3$ and for any given $p>2^{*}=\frac{2 n}{n-2}$ we consider solutions $u=u(r)$ of the equation

$$
\left.u_{r r}+\frac{n-1}{r} u_{r}+|u|^{p-2} u=0 \text { on }\right] 0, \infty[
$$

corresponding to (1.1). In our first result we study entire solutions $u \in C^{2}([0, \infty[)$ of (2.1) with a well-defined limit

$$
u(0)=u_{0}, u_{r}(0)=0 \text { at } r=0,
$$

giving rise to classical solutions of (1.1) on $\mathbb{R}^{n}$. Moreover, we prove that the function $u^{*}$ defined in (1.5) is the only radial solution of (1.1) with a mild singularity at the origin.

Theorem 2.1. i) Let $u \in C^{2}\left(\left[0, \infty[)\right.\right.$ solve (2.1), (2.2) for some $u_{0}>0$. Then $u(r)>0$, and $u^{\prime}(r)<0$ for $r>0$. Finally, there holds

$$
r^{a} u(r) \rightarrow b^{\frac{1}{p-2}} \text { as } r \rightarrow \infty,
$$

for $a=\frac{2}{p-2}$ and $b=a(n-a-2)$ as above.

ii) Suppose that $u \in C^{2}(] 0, \infty[)$ solves (2.1) with

$$
\liminf _{r \rightarrow 0} r^{\frac{2 p}{p-2}}\left(\left|u_{r}\right|^{2}+|u|^{p}\right)<\infty .
$$

Then either $u \in C^{2}\left([0, \infty[)\right.$, and $u$ is as in $i)$, or $u= \pm u^{*}$.

From Theorem 2.1.i) we immediately obtain the characterization $u^{*}=\lim _{R \rightarrow \infty} u_{R}$ for any solution $0<u \in C^{2}([0, \infty[)$ of $(1.1)$.

Note that the regular solutions of (2.1) are uniquely determined by the choice of $u_{0}$ in (2.2). Using (1.2) and invariance of (1.1) under reflection $u \rightarrow-u$ they can be obtained by rescaling the solution satisfying $(2.2)$ with initial data $u_{0}=1$.

Before giving the proof of Theorem 2.1, we first remark that for equation (2.1) the classical Pohozaev identity [7] takes the following form.

Theorem 2.2. Let $u \in C^{2}(] \rho, R[)$ be a solution of equation (2.1). Then we have

$$
\left.\frac{d}{d r}\left[r^{n}\left(\frac{1}{2}\left|u_{r}\right|^{2}+\frac{1}{p}|u|^{p}+\frac{n-2}{2 r} u_{r} u\right)\right]+\left(\frac{n-2}{2}-\frac{n}{p}\right) r^{n-1}|u|^{p}=0 \text { in }\right] \rho, R[.
$$


A similarly useful identity results if we consider equation (2.1) in scaled coordinates. Introducing $s=\log r$, we define $v(s)=r^{a} u(r)$ so that $u(r)=e^{-a s} v(s)$. Compute

$$
\begin{aligned}
r^{a+1} u_{r}(r) & =v^{\prime}(s)-a v(s), \\
r^{a+2} u_{r r}(r) & =v^{\prime \prime}(s)-(2 a+1) v^{\prime}(s)+a(a+1) v(s) .
\end{aligned}
$$

Hence (2.1) translates into the equation

$$
v^{\prime \prime}(s)+\mu v^{\prime}(s)-b \cdot v(s)+|v|^{p-2} v(s)=r^{a+2}\left(u_{r r}+\frac{n-1}{r} u_{r}+|u|^{p-2} u\right)=0
$$

for $v$, with $\mu=n-\frac{2 p}{p-2}=n-2-2 a$ and $b=a(n-2-a)=a(\mu+a)$ as defined above. Note that $\mu>0$ if (and only if) $p>2^{*}$.

Multiplying (2.5) with $2 v^{\prime}$ we find the identity

$$
\frac{d}{d s}\left(\left|v^{\prime}\right|^{2}-b v^{2}+\frac{2}{p}|v|^{p}\right)+2 \mu\left|v^{\prime}\right|^{2}=0 .
$$

For later reference, for a solution $v$ to $(2.5)$ we define

$$
g(s)=\left(\left|v^{\prime}\right|^{2}-b v^{2}+\frac{2}{p}|v|^{p}\right)(s) .
$$

By (2.6) then for any such $v$ the function $s \mapsto g(s)$ is non-increasing. Moreover, $g \geq-\frac{p-2}{p} b^{\frac{p}{p-2}}$ is uniformly bounded from below; we remark that this lower bound is achieved for the constant solution $v \equiv b^{\frac{1}{p-2}}$ of (2.5) which represents the selfsimilar solution $u^{*}$ of (2.1). We furthermore remark that solutions of (2.1) satisfying the assumption (2.3) of Theorem 2.1.ii) correspond exactly to those solutions for which $g$ is bounded.

Proof of Theorem 2.1. i) As observed earlier, it suffices to consider the case $u_{0}=1$. Integrating (2.4) from $\rho=0$ to $r$, we find that $u_{r} u<0$ on ]0, $\infty[$. Since $u(0)=1>0$ it follows that $u(r)>0, u_{r}(r)<0$ for $r>0$.

Next, from (2.2) we see that the corresponding function $v(s)=r^{a} u(r) \rightarrow 0$ and $v^{\prime}(s) \rightarrow 0$ as $s \rightarrow-\infty$ so

$$
C_{-\infty}:=\lim _{s \rightarrow-\infty} g(s)=0 .
$$

Recalling that the quantity $g$ defined in (2.7) is non-increasing and bounded from below we conclude that also the limit

$$
C_{\infty}:=\lim _{s \rightarrow \infty} g(s)=\lim _{s \rightarrow \infty}\left(\left|v^{\prime}\right|^{2}-b v^{2}+\frac{2}{p} v^{p}\right) \leq 0
$$

exists; moreover, (2.6) yields that $v^{\prime} \in L^{2}(\mathbb{R})$ and

$$
C_{\infty}+2 \mu \int_{-\infty}^{\infty}\left|v^{\prime}\right|^{2} d s=C_{-\infty}
$$

It then follows that

$$
\lim _{s \rightarrow \infty} v^{\prime}(s)=0, \lim _{s \rightarrow \infty}\left(\frac{2}{p} v^{p}-b v^{2}\right)=C_{\infty},
$$

because for any $\epsilon>0$ by (2.10) we have $\inf _{[s, s+\epsilon]}\left|v^{\prime}\right|^{2} \rightarrow 0(s \rightarrow \infty)$, and (2.5), (2.9) limit the rate of change of $v^{\prime}$. But (2.11) implies convergence $v(s) \rightarrow v_{\infty}$ 
as $s \rightarrow \infty$; moreover, together with (2.5) the relation (2.11) yields that also $v^{\prime \prime}(s)$ converges to a limit which must be zero as $v^{\prime}$ converges. Therefore

$$
-b v+v^{p-1} \rightarrow 0 \text { as } s \rightarrow \infty
$$

and we conclude that either $v_{\infty}=0$ and $C_{\infty}=0$, or $v_{\infty}=b^{\frac{1}{p-2}}$ and $C_{\infty}=$ $-\frac{p-2}{p} b^{\frac{p}{p-2}}=\min g$.

But if $C_{\infty}=0$, from (2.8) and (2.10) we conclude that $v^{\prime} \equiv 0$ and hence $v \equiv$ $\lim _{s \rightarrow \infty} v(s)=0$, which implies that also $u \equiv 0$ and thus contradicts our assumption that $u(0)=1$. Therefore we must have $v(s) \rightarrow b^{\frac{1}{p-2}}$ as $s \rightarrow \infty$, that is, $r^{a} u(r) \rightarrow$ $b^{\frac{1}{p-2}}(r \rightarrow \infty)$, as claimed.

ii) Observe the relation $b-a^{2}=a(n-2-2 a)=a \mu>0$. Thus, by Young's inequality we can estimate

$$
\left|v^{\prime}\right|^{2}-b v^{2}=\left(r^{a+1} u_{r}+a \cdot r^{a} u\right)^{2}-b r^{2 a} u^{2} \leq C r^{2(a+1)} u_{r}^{2}=C r^{\frac{2 p}{p-2}} u_{r}^{2}
$$

with a constant $C=C(p, n)>0$. If now we assume that

$$
C_{-\infty}:=\lim _{s \rightarrow-\infty}\left(\left|v^{\prime}\right|^{2}-b v^{2}+\frac{2}{p}|v|^{p}\right) \leq C \liminf _{r \rightarrow 0} r^{\frac{2 p}{p-2}}\left(\left|u_{r}\right|^{2}+|u|^{p}\right)<\infty,
$$

similar to the above we conclude that $v^{\prime}(s), v^{\prime \prime}(s) \rightarrow 0$ as $s \rightarrow-\infty$, and either $v(s) \rightarrow \pm b^{\frac{1}{p-2}}$ as $s \rightarrow-\infty$ and $C_{-\infty}=\min g$, or $v(s) \rightarrow 0$ as $s \rightarrow-\infty$. In the latter case it follows that $r^{a} u(r), r^{a+1} u_{r}(r) \rightarrow 0$ as $r \rightarrow 0$; in particular,

$$
\frac{1}{r^{\mu}} \int_{B_{r}(0)}\left(\left|u_{r}\right|^{2}+|u|^{p}\right) d x \rightarrow 0 \text { as } r \rightarrow 0,
$$

since $\mu=n-2-2 a=n-\frac{2 p}{p-2}=n-a p$. From Pacard's [6] Proposition 1 and Lemma 8 then we obtain regularity for $u$ at $x=0$, so $u$ must be as in $\mathrm{i}$ ). (As observed in [1], any $p>2^{*}$ is admissible for Pacard's result.)

Thus we may assume that $C_{-\infty}=\min g$ and thus that $s \mapsto g(s)$ is constant. It follows that $v \equiv \lim _{s \rightarrow-\infty} v(s)= \pm b^{\frac{1}{p-2}}$, and $u= \pm u^{*}$.

From Theorem 2.1 we know that any solution $u \in C^{2}(] 0, \infty[)$ of $(2.1)$ that vanishes at some point $R>0$ must be strongly singular at $x=0$. In fact, we show in the next result that also the converse is true. Furthermore we obtain that statement ii) of the previous result remains valid under much weaker regularity assumptions.

Theorem 2.3. Suppose that $u \in C^{2}(] 0, \infty[)$ solves equation (2.1) with

$$
\liminf _{r \rightarrow 0} r^{\frac{2 p}{p-2}}\left(\left|u_{r}\right|^{2}+|u|^{p}\right)=\infty \text {. }
$$

Then $u$ changes sign infinitely often on any interval $] 0, R[, R>0$, and there exists a number $c_{0}>0$ such that

$$
\left|u_{r}\right|^{2}+\frac{2}{p}|u|^{p}=c_{0} r^{-(n+d)}+O\left(r^{-n}\right) \text { as } r \rightarrow 0,
$$

where $d=\frac{2 p}{p+2}(n-1)-n>0$.

Since $n+d>n>\frac{2 p}{p-2}$ for $p>2^{*}$, we thus see that the only radially symmetric solutions of (1.1) that are locally in $H^{1}$ are the selfsimilar solutions $\pm u^{*}$ and the regular solutions $u \in C^{2}([0, \infty[)$ described in Theorem 2.1. 
Proof. i) Let $u$ be as above. For brevity in the following we use the shorthand notation $u^{\prime}=u_{r}$ also with reference to $r$. Consider the quantity

$$
\Phi_{0}(r):=r^{n}\left(\left|u^{\prime}\right|^{2}+\frac{2}{p}|u|^{p}\right)
$$

from Theorem 2.2 which satisfies

$$
\Phi_{0}^{\prime}(r)=n r^{-1} \Phi_{0}(r)+2 r^{n}\left(u^{\prime \prime}+|u|^{p-2} u\right) \cdot u^{\prime}=n r^{-1} \Phi_{0}(r)-2(n-1)\left|u^{\prime}\right|^{2} \cdot r^{n-1} .
$$

While not monotone itself, a slight modification of $\Phi_{0}$ similar to (2.4) will give us a monotone quantity that can be used to study the asymptotic behaviour of $u$ near zero.

Writing equation (2.1) in divergence form

$$
\left(r^{n-1} u^{\prime}\right)^{\prime}=-r^{n-1}|u|^{p-2} u
$$

and letting

$$
\Phi(r):=\Phi_{0}(r)+c \cdot r^{n-1} u^{\prime} \cdot u,
$$

with a constant $c \in \mathbb{R}$ to be determined, we find

$$
\begin{aligned}
\Phi^{\prime}(r) & =\Phi_{0}^{\prime}(r)+c r^{n-1}\left(\left|u^{\prime}\right|^{2}-|u|^{p}\right) \\
& =n r^{-1} \Phi_{0}-\left[(2 n-2-c)\left|u^{\prime}\right|^{2}+\frac{c p}{2} \cdot \frac{2}{p}|u|^{p}\right] r^{n-1} .
\end{aligned}
$$

For $c=\frac{4}{2+p}(n-1)$ chosen so that $(2 n-2-c)=\frac{c p}{2}$ we then obtain the identity

$$
\Phi^{\prime}(r)=-d r^{-1} \Phi_{0}(r)
$$

for the positive number $d$ defined in the statement of Theorem 2.3.

Remark that for solutions of (2.1) satisfying (2.13) the difference $\Phi-\Phi_{0}$ is small compared with $\Phi$. Indeed, for any number $\varepsilon \in] 0,1[$ we may estimate

$$
\left|\Phi-\Phi_{0}\right| \leq C \Phi_{0}^{\frac{2+p}{2 p}} r^{n-1-\frac{2+p}{2 p} \cdot n}=C\left(r^{d} \Phi_{0}\right)^{\frac{2+p}{2 p}} \leq \frac{1}{8} \varepsilon \Phi_{0}+C_{\varepsilon} r^{\mu},
$$

so that by assumption (2.13)

$$
\left|\Phi-\Phi_{0}\right| \leq \frac{1}{4} \varepsilon \Phi_{0} \leq \frac{\varepsilon}{4-\varepsilon} \Phi
$$

for sufficiently small $r>0$. Hence we also have

$$
\left|\Phi-\Phi_{0}\right| \leq \frac{\varepsilon}{d} \Phi
$$

for $0<r<r_{\varepsilon}$ small enough. Equation (2.16) thus gives the differential inequality

$$
-(d+\varepsilon) r^{-1} \Phi(r) \leq \Phi^{\prime}(r) \leq-(d-\varepsilon) r^{-1} \Phi(r)
$$

which results in estimates of the form

$$
C_{1} \cdot r^{-d+\varepsilon} \leq \Phi(r), \Phi_{0}(r) \leq C_{2} \cdot r^{-d-\varepsilon},
$$

on ]0, $r_{\varepsilon}\left[\right.$, with numbers $C_{1,2}>0$ depending on $\varepsilon$.

Inserting the bound (2.19) in (2.17) we can now improve (2.18) to obtain

$$
\left|\Phi-\Phi_{0}\right| \leq C\left(r^{d} \Phi_{0}\right)^{\frac{2+p}{2 p}} \leq C r^{-\frac{2+p}{2 p} \varepsilon} \leq C r^{-\varepsilon} .
$$

This bound in turn gives the improved differential inequality

$$
\left.\left|\left(r^{d} \Phi(r)\right)^{\prime}\right|=r^{d}\left|\Phi^{\prime}+d r^{-1} \Phi\right|=d r^{d-1}\left|\Phi-\Phi_{0}\right| \leq C r^{d-1-\varepsilon} \text { on }\right] 0, r_{\varepsilon}[.
$$


Integrating this inequality for a fixed number $\varepsilon \in] 0, d[$ we conclude that there exists

$$
c_{0}=\lim _{r \rightarrow 0} r^{d} \Phi(r)=\lim _{r \rightarrow 0} r^{d} \Phi_{0}(r) .
$$

By (2.17) then $\left|\Phi-\Phi_{0}\right| \leq C$, and we find the improved bound $\left|\left(r^{d} \Phi(r)\right)^{\prime}\right| \leq C r^{d-1}$ from which we obtain that

$$
\left|r^{d} \Phi_{0}(r)-c_{0}\right| \leq C r^{d}+\left|r^{d} \Phi(r)-c_{0}\right| \leq C r^{d},
$$

as claimed. Observe that the limit $c_{0}$ must be positive; otherwise estimate (2.19) would be violated.

ii) In a second step we now show that any solution $u$ of (2.1) satisfying (2.13), and therefore also satisfying (2.14), changes sign infinitely often as $r \downarrow 0$. Assume by contradiction that some such $u$ does not change sign on an interval $] 0, r_{1}[$ for some $r_{1}>0$, say $u(r) \geq 0$ for $0<r<r_{1}$.

We first claim that $u$ must be decreasing in $] 0, r_{1}[$. Indeed, since the maximum principle implies that solutions of (2.1) cannot achieve non-negative local minima, the only alternative would be that $u$ is increasing on an interval $] 0, r_{2}[$ for some $0<r_{2}<r_{1}$. However the resulting bound $0 \leq u \leq u\left(r_{2}\right)$ and the asymptotics of $\Phi_{0}$ shown above then imply that $u^{\prime}(r) \geq c \cdot r^{-\frac{n+d}{2}}$ as $r \rightarrow 0$, which contradicts our assumption that $u \geq 0$.

Now recall that $c_{0}=\lim _{r \rightarrow 0} \Phi_{0}(r) r^{d}>0$ and consider the sets

$$
\begin{aligned}
& U_{1}(R):=\left\{r \in[R, 2 R] ;\left|u^{\prime}(r)\right|^{2} \geq \frac{1}{4} c_{0} r^{-n-d}\right\}, \\
& U_{2}(R):=\left\{r \in[R, 2 R] ; \frac{2}{p} u(r)^{p} \geq \frac{1}{4} c_{0} r^{-n-d}\right\},
\end{aligned}
$$

whose union contains the whole interval $[R, 2 R]$, provided $R>0$ is sufficiently small.

Since $u^{\prime} \leq 0$ and $u>0$ near zero, for the 1-dimensional Lebesgue measure $\mu\left(U_{1}(R)\right)$ we obtain the inequality

$$
u(R) \geq-\int_{R}^{2 R} u^{\prime}(r) d r \geq c_{1} R^{-(n+d) / 2} \cdot \mu\left(U_{1}(R)\right),
$$

with a constant $c_{1}>0$ depending on $c_{0}$ but independent of $R$. In view of the estimate

$$
u(R) \leq C\left(R^{-n} \Phi_{0}(R)\right)^{1 / p} \leq C R^{-(n+d) / p}
$$

we then find the upper bound

$$
\mu\left(U_{1}(R)\right) \leq C R^{\gamma}, \text { with } \gamma=(n+d) \cdot \frac{p-2}{2 p}>1 .
$$

On the other hand, upon integrating equation (2.15) we find that

$$
-\int_{0}^{2 R}\left(u^{\prime} r^{n-1}\right)^{\prime} d r=\int_{0}^{2 R} u^{p-1} r^{n-1} d r \geq c R^{n-1-(n+d) \cdot \frac{p-1}{p}} \mu\left(U_{2}(R)\right) .
$$

Since $u^{\prime}(r) r^{n-1} \rightarrow 0$ as $r \rightarrow 0$ the left hand side is equal to $-u^{\prime}(2 R)(2 R)^{n-1}$ and thus bounded from above by $C \cdot R^{n-1-(n+d) / 2}$. We conclude that also the measure of the second set is bounded by $\mu\left(U_{2}(R)\right) \leq C R^{\gamma}$ and thus in total

$$
\mu\left(U_{1}(R)\right)+\mu\left(U_{2}(R)\right) \leq C R^{\gamma}<<R=\mu([R, 2 R])
$$

for small $R>0$, which contradicts the fact that $[R, 2 R] \subset U_{1}(R) \cup U_{2}(R)$. 
Thus, sign-changing solutions are necessarily oscillatory near the origin. On the other hand, we now show that the sign of any solution to (2.1) stabilizes near infinity.

Theorem 2.4. Let $u \in C^{2}(] 0, \infty[)$ solve (2.1). Then there exists $R>0$ such that $u(r) \neq 0$ for $r>R$, and either $\frac{u(r)}{u^{*}(r)} \rightarrow \pm 1$ as $r \rightarrow \infty$, or there exists a number $c_{1} \neq 0$ such that

$$
u(r)=r^{-(n-2)}\left(c_{1}+O\left(r^{-(2+\mu(p-2))}\right) .\right.
$$

Proof. Let $v(s)=r^{a} u(r)$ be defined as above, satisfying (2.5). As in the proof of Theorem 2.2, from (2.6) we conclude that the limit

$$
C_{\infty}:=\lim _{s \rightarrow \infty} g(s)=\lim _{s \rightarrow \infty}\left(\left|v^{\prime}\right|^{2}-b v^{2}+\frac{2}{p}|v|^{p}\right)<\infty
$$

exists and that $v^{\prime} \in L^{2}([0, \infty[)$ with

$$
C_{\infty}+2 \mu \int_{0}^{\infty}\left|v^{\prime}\right|^{2} d s=g(0)<\infty
$$

Still following the proof of Theorem 2.2, we then conclude that

$$
v^{\prime}(s) \rightarrow 0 \text { as } s \rightarrow \infty,
$$

and either $v(s) \rightarrow 0$ as $s \rightarrow \infty$, or $v(s) \rightarrow \pm b^{\frac{1}{p-2}}$. In the latter case, $\frac{u(r)}{u^{*}(r)} \rightarrow$ \pm 1 and clearly our claim is true. In the following we therefore may assume that $\lim _{s \rightarrow \infty} v(s)=0$.

Let now $s_{1} \in \mathbb{R}$ be such that $|v|^{p-2}<b$ for $s>s_{1}$. Then on $\left[s_{1}, \infty\right)$ the expression $v^{\prime \prime}(s)+\mu v^{\prime}(s)=\left(b-|v|^{p-2}\right) v$ has the same sign as $v(s)$, so $v$ can neither achieve a positive maximum nor a negative minimum on $\left[s_{1}, \infty\right)$. So if there is any point $s_{0}>s_{1}$ with $v\left(s_{0}\right)=0$ we thus find that $v$ is monotone on $\left[s_{0}, \infty\right)$; in particular, $v(s) \neq 0$ for $s>s_{0}$. If no such $s_{0}$ exists we define $s_{0}:=s_{1}$ so that again $v(s) \neq 0$ for $s>s_{0}$.

To see also the claimed asymptotic behavior, for $s>s_{0}$ we divide (2.5) by $v$ to find the identity

$$
\left(\frac{v^{\prime}}{v}\right)^{\prime}+\left(\frac{v^{\prime}}{v}\right)^{2}+\mu \frac{v^{\prime}}{v}=\frac{v^{\prime \prime}}{v}+\mu \frac{v^{\prime}}{v}=b-|v|^{p-2}
$$

for $b=a(\mu+a)$ as above.

For $f:=v^{\prime} / v$ this gives the equation

$$
f^{\prime}+f^{2}+\mu f-b=f^{\prime}+(f-a)(f+\mu+a)=-|v|^{p-2} .
$$

We claim that $\lim _{s \rightarrow \infty} f(s)=-(\mu+a)$. First of all, if $f\left(s_{2}\right)<-(\mu+a)$ for some $s_{2}$ then $f$ would be decreasing on $\left[s_{2}, \infty\right)$ and thus satisfy $f^{\prime}(s) \leq-\varepsilon f^{2}(s)$ for some $\varepsilon>0$ so that $f(s) \rightarrow-\infty$ as $s \rightarrow s_{-}$for some $s_{-}<\infty$ which is impossible as $v(s) \neq 0$ for $s>s_{0}$.

On the other hand, inspection of the sign of the nonlinearity combined with the assumption that $v(s) \rightarrow 0$ shows that if $\limsup _{s \rightarrow \infty} f(s)>-(\mu+a)$ then $f$ tends to $a=\frac{2}{p-2}$ as $s \rightarrow \infty$. But then $v^{\prime} \geq v /(p-2)$ for large $s$, and $v(s) \rightarrow \infty$ as $s \rightarrow \infty$, contrary to our assumption. We thus conclude that $f(s) \rightarrow-(\mu+a)$ as $s \rightarrow \infty$.

Given any $\epsilon>0$ we then find that for sufficiently large $s>s_{0}(\epsilon)$

$$
-(\mu+a) \leq \frac{v^{\prime}}{v} \leq(-\mu+a+\varepsilon)
$$


which gives

$$
v\left(s_{0}\right) e^{-(\mu+a)\left(s-s_{0}\right)} \leq v(s) \leq v\left(s_{0}\right) e^{-(\mu+a-\varepsilon)\left(s-s_{0}\right)} .
$$

as a first estimate on the asymptotics of $v$. Since $\mu+2 a=n-2$ this translates into the bound

$$
u\left(r_{0}\right)\left(r / r_{0}\right)^{-(n-2)} \leq u(r) \leq u\left(r_{0}\right)\left(r / r_{0}\right)^{-(n-2-\epsilon)}
$$

for sufficiently large $r>r_{0}(\epsilon)$.

To obtain the more precise asymptotic expansion claimed in Theorem 2.4 we now consider the function $h(s):=f(s)+\mu+a=f(s)+n-2>0$ which satisfies

$$
h^{\prime}-(n-2) h=-h^{2}-|v|^{p-2} \geq-\varepsilon h-|v|^{p-2}
$$

for $r \geq r_{0}(\varepsilon)$ sufficiently large. Inserting the bound on $v$ derived in (2.26) we find that

$$
e^{(n-2-\varepsilon) s} \cdot\left(h(s) \cdot e^{-(n-2-\varepsilon) s}\right)^{\prime} \geq-c e^{-(p-2)(\mu+a-\varepsilon) s}
$$

for $s$ large. Since $h(s) \rightarrow 0$ as $s \rightarrow \infty$ this gives the estimate

$$
h(s) \leq C e^{-(p-2)(\mu+a-\varepsilon) s}
$$

which in turn allows us to improve the differential inequality (2.25) for $v$ to

$$
0 \leq v^{\prime}+(\mu+a) v \leq v h \leq C e^{-(p-1)(\mu+a-\varepsilon) s}
$$

so that

$$
\left(e^{(\mu+a) s} v(s)\right)^{\prime} \leq C e^{-(p-2)(\mu+a-\varepsilon) s} .
$$

Since $p>2$ we conclude that the limit

$$
c_{1}=\lim _{s \rightarrow \infty} e^{(\mu+a) s} v(s)=\lim _{r \rightarrow \infty} r^{n-2} u(r)
$$

exists, and thus

$$
v(s) \leq C e^{-(\mu+a) s} \text { for large } s .
$$

Repeating the above argument with the bound

$$
\begin{aligned}
h^{\prime}-(n-2) h & =-h^{2}-|v|^{p-2} \geq-C e^{-2(p-2)(\mu+a-\varepsilon) s}-C e^{-(p-2)(\mu+a) s} \\
& \geq-C e^{-(p-2)(\mu+a) s}, s>>1,
\end{aligned}
$$

resulting from (2.30) and (2.31) we obtain the estimate claimed in (2.22).

\section{Self-Similar solutions}

Recall that a solution $u$ of (1.1) on $\mathbb{R}^{n} \backslash\{0\}$ is self-similar if

$$
u(x)=u_{R}(x)=R^{a} u(R x) \text { for all } x \neq 0 \text { and any } R>0 .
$$

As we have seen in Section 2, equation (1.1) admits the self-similar radial solution $u^{*}$, and $u^{*} \in H_{l o c}^{1} \cap L_{l o c}^{p}\left(\mathbb{R}^{n}\right)$ when $p>2^{*}$. By Pacard's work [6] we also expect to find not necessarily radial self-similar weak solutions of (1.1) on $\mathbb{R}^{n}$ as limits of blow-ups of singular "stationary" weak solutions $u$ of equation (1.1) on a domain, and the question of classifying these singularity profiles is of interest. Self-similar solutions to (1.1) also may arise as asymptotic profiles of stationary solutions at infinity, as described in our paper [8], or as singularity profiles of solutions of the parabolic evolution problem associated with (1.1) at blow-up points of Type I, as defined in the forthcoming work [1] of Simon Blatt and the second author, which extends the work of Giga-Kohn [3] to the critical and super-critical range and also 
removes the restriction on the range of admissible exponents $p$ in the results of Pacard [6].

Differentiating relation (3.1) in $R$ for fixed $x \in S^{n-1}=\left\{x \in \mathbb{R}^{n} ;|x|=1\right\}$, we find the relations

$$
u_{r}+a u / r=0, u_{r r}=a(a+1) u / r^{2} \text { for } r=|x|>0 .
$$

Moreover choosing $R=|x|^{-1}$ for each $x \neq 0$, we obtain the representation (1.3) with a function $v: S^{n-1} \rightarrow \mathbb{R}$.

Thus, evaluating (1.1) at a point $x \in S^{n-1}$, from (3.2) we obtain the equation

$$
\begin{aligned}
0 & =\Delta u+|u|^{p-2} u=u_{r r}+(n-1) u_{r}+\Delta_{S^{n-1}} u+|u|^{p-2} u \\
& =\Delta_{S^{n-1}} v-a(n-2-a) v+|v|^{p-2} v ;
\end{aligned}
$$

that is,

$$
-\Delta_{S^{n-1}} v+b \cdot v=|v|^{p-2} v \text { on } S^{n-1},
$$

or (1.4). We conclude this paper with the following remarks characterizing the solutions of $(3.3)$ in the range $2^{*}<p \leq 2^{+}:=\frac{2(n-1)}{n-3}$, the critical exponent in $n-1$ dimensions (which almost equals the range covered by Pacard's [6] results). Observe that by standard regularity results for $p \leq 2^{+}$any solution $v \in H^{1}$ of $(3.3)$ is smooth.

By using the method of Ding [2] one can show that for any $p<\frac{2 m}{m-2}$, where $n=m+k$ with $2 \leq k \leq m$, equation (3.3) possesses infinitely many distinct solutions of changing sign, giving rise to infinitely many distinct sign-changing solutions of (1.1) which blow up at the origin at the rate $|x|^{-a}$. Choosing the minimal number $m$ with the above property, for even dimensions $n$ we have $m=\frac{n}{2}$ and $\frac{2 m}{m-2}=\frac{2 n}{n-4}>2^{+}$; likewise $\frac{2 m}{m-2}=\frac{2(n+1)}{n-3}>2^{+}$when $n=2 m-1$ is odd. Thus, in general there is a huge multitude of self-similar solutions of (1.1).

However, the picture changes if we restrict our attention to positive solutions. In fact, by a result of Gidas and Spruck [4] for $p<2^{+}$any positive solution of (3.3) is constant. Indeed, even though for $p<2^{+}$we have $b>\frac{(n-1)(n-3)}{4}$ while there holds $R_{i j}=(n-2) g_{i j}$ for the Ricci curvature of $S^{n-1}$ in the standard metric $g=\left(g_{i j}\right)$, the condition (B.10) in the Remark following Theorem B.2 in [4] is satisfied for the number $\gamma>0$ given by (B.11), and the conclusion follows from [4], Theorem B.2.

Finally, in the critical case $p=2^{+}=\frac{n-1}{n-3}$ we have

$$
b=\frac{(n-1)(n-3)}{4}=\frac{1}{c(n-1)} R_{g_{S^{n-1}}}
$$

with $R_{h}$ the scalar curvature and $c(k)=4 \frac{k-1}{k-2}$ the constant appearing in the conformal Laplace operator

$$
L_{h}=-c(k) \Delta_{h}+R_{h}
$$

on a $k$-dimensional manifold $(M, h)$, and $(3.3)$ is the Yamabe equation on $S^{n-1}$, with infinitely many positive solutions $u$ exactly arising as the coefficient in the conformal factor $u^{\frac{4}{n-3}} g_{S^{n-1}}$ of the metric obtained by pulling back the standard spherical metric $g_{S^{n-1}}$ with a Möbius transformation; see Obata [5].

In a sequel [8] to this paper we plan to investigate (1.4) also in the super-critical range $p>2^{+}$and, in particular, construct weak solutions $0<v \in H^{1}$ depending only on the distance $\rho(q)=\operatorname{dist}(q, N)$ of the point $q \in S^{n-1}$ from the North pole $N$ and blowing up at the rate $v(\rho) \sim \rho^{-a}$ as $\rho \rightarrow 0$, similar to $u^{*}$. 


\section{REFERENCES}

[1] Blatt, S.; Struwe, M.: Monotonicity and blow-up for a supercritical semilinear heat equation, in preparation.

[2] Ding, Wei Yue: On a conformally invariant elliptic equation on $\mathbb{R}^{n}$, Comm. Math. Phys. 107 (1986), no. 2, 331-335.

[3] Giga, Yoshikazu; Kohn, Robert V.: Asymptotically self-similar blow-up of semilinear heat equations, Comm. Pure Appl. Math. 38 (1985), no. 3, 297319.

[4] Gidas, B.; Spruck, J.: Global and local behavior of positive solutions of nonlinear elliptic equations, Comm. Pure Appl. Math. 34 (1981), no. 4, 525-598.

[5] Obata, Morio: The conjectures on conformal transformations of Riemannian manifolds, J. Differential Geometry 6 (1971/72), 247258.

[6] Pacard, Frank: Partial regularity for weak solutions of a nonlinear elliptic equation, Manuscripta Math. 79 (1993), no. 2, 161-172.

[7] Pohozaev, S. I.: On the eigenfunctions of the equation $\Delta u+\lambda f(u)=0$, Dokl. Akad. Nauk SSSR 1651965 36-39.

[8] Rupflin, M.; Struwe, M.: Self-similar solutions of a supercritical equation of Yamabe-type, in preparation.

(Melanie Rupflin) Max-Planck Institute for Graviational Physics, Am Mühlenberg 1, D-14485 PotsDAm

E-mail address: melanie.rupflin@aei.mpg.de

(Michael Struwe) Departement Mathematik, ETH-Zürich, CH-8092 Zürich

E-mail address: michael.struwe@math.ethz.ch 